\title{
Bacterial Extracts as Immunomodulators for the Prevention of Recurrent Respiratory Infections in Children
}

\section{Giovannini M, Salvini F* and Riva E}

Pediatric Department University of Milan, San Paolo Hospital - Milan, Italy

*Corresponding author: Filippo Salvini, Pediatric Department University of Milan, San Paolo Hospital, Via Di Rudinì, 820142 , Milan-Italy, Tel: +39 02 8184 4666; Fax: +3902 8184 3810; E-mail: filippo.salvini@ao-sanpaolo.it

Rec Date: Dec 23, 2013, Acc date: May 30, 2014, Pub date: June 4, 2014

Copyright: (c) 2014 Giovannini M, et al. This is an open-access article distributed under the terms of the Creative Commons Attribution License, which permits unrestricted use, distribution, and reproduction in any medium, provided the original author and source are credited.

\begin{abstract}
Acute respiratory tract infections (ARTI) are the more frequent causes of morbidity and mortality in children. This review was conducted to assess the existing evidence concerning bacterial extracts efficacy in the prevention of pediatric ARTI.
\end{abstract}

The data sources for the identification of clinical trials and reviews included principal bibliographic databases (MEDLINE, Embase, Cochrane Library, PubMed/www.ncbi.nlm.nih.gov). Selected clinical trials only involved children suffering from recurrent ARTI.

Bacterial extracts may reduce the incidence of ARTI of about $40 \%$ in toddlers (2-5 years), school boys (6-12 years) and children at higher ARTI risk (e.g. children living in orphanages). Among others, the active treatment with OM-85 led to $26,2 \%$ fewer patients with recurrent ARTI. Data from the literature are encouraging, particularly for treatment with OM-85.

The strengthening of pre-clinical and clinical research is necessary to allow routine recommendation of bacterial extracts prescription for ARTI prevention in children.

Keywords: Bacterial extracts; Immunomodulators; Children

\section{Introduction}

Acute respiratory tract infections (ARTI: acute nasopharyngitis, sinusitis, acute otitis, tonsillitis, viral croup, or pneumonia) are characterized by the onset of more than six respiratory infections per year or more than one upper respiratory infection per month or more than three lower respiratory infections during the period of maximum exposure (September to April), in a child that does not suffer pathological conditions to justify respiratory infections recurrence, such as primary immunodeficiencies, HIV infection, cystic fibrosis, immotile cilia syndrome or congenital abnormalities [1]. In western countries ARTI occurs in $25 \%$ of children within the first year of life and in $18 \%$ of children in the age group of 1-4 years [2].

ARTI are a common problem mainly in pre-school age, due to the presence of unfavourable environmental conditions, including early socialization, as well as the immaturity and inexperience of the immune system.

In particular, immunological immaturity that characterizes the preschool years makes this age most exposed to ARTI. Though it is a benign condition, destined to evolve favorably around 12 years of life, ARTI represent a health care system, as they interfere with the child's welfare and lead to important medical-legal costs [2].

It resulted therefore crucial to attend preventive mechanisms in pre-school age.
In $80 \%$ of cases these infections are caused by rhinovirus, parainfluenza and influenza viruses and the remaining $20 \%$ are due to $\beta$-hemolytic streptococcus group A.

The risk factors are mostly environmental, such as early socialization, passive smoking and pollution exposure; the small airway caliber and the difficulty to blow the nose or cough up secretions helps to increase the risk of contracting respiratory infections in infants and toddlers.

In the child suffering ARTI some alterations in load of one or more elements of the immune response are detectable. The occurrence of these changes in the immune response appears to be due to the negative action that many viruses and some bacterial agents can play against the lymphocyte and granulocyte function. These changes are, however, mild and transient, and there seems no immunological disregulation that can be defined as typical of the child suffering from ARTI [1].

The immune defects in children with recurrent respiratory infections have been frequently reported in the literature and include altered $\mathrm{T}$ Lymphocytes helper/suppressor ratio in 50\% of cases, reduced T-lymphocyte response to mitogens such as PHA (phytohemagglutinin) in $30 \%$, reduced chemotactic and phagocytic activity of neutrophils in $66-100 \%$ of cases and impaired NK cell function in another $30 \%$ [3].

Although falling under physiological conditions, the relapse of infections is a cause of concern for families and a relevant public health issue. In addition to the management of the individual episodes, the major interest is to prevent and positively influence the natural 
history of ARTI. Approaching this kind of patients, it is also necessary to exclude adverse pathological factors such as allergy, asthma and adeno-tonsillar hypertrophy.

It should be avoided, at least temporarily, environmental factors, such as the frequency in child community, which facilitate the onset and relapse of respiratory infections.

Patients presenting with greater clinical severity could benefit by some simple diagnostic tests: complete blood count with leucocytes differential count, immunoglobulin levels, protein electrophoresis (in order to detect $\alpha$-1-antitrypsin defect, which is associated with reduced a-1-globulin levels), sweat testing (recommended in recurrent lower respiratory tract infections).

Adenoidectomy is recommended for recurrent otitis if adenoidal hypertrophy is documented. Tonsillectomy can also be indicated in relapsing tonsillitis.

Anti-pneumococcal [4] and anti-influenza vaccinations [5] also play an important role in the prevention of ARTI.

Actually, there is increasing interest on the clinical efficacy of biological immune response modifiers, arguing that these could lead to a better immune response and therefore better clinical outcome, with shorter duration of symptoms, reduction in the number of antibiotic treatments and fewer days lost from kindergarten/school. These products are expected to correct subtle immune defects observed in children with ARTI (e.g. deficient chemotaxis of granulocytes, alterations in lymphocyte subpopulations) [6-9].

The main objective of this review was to examine the effectiveness of the more studied bacterial extracts available on the market to steer its use in clinical practice.

The data sources for the identification of trials and reviews included principal bibliografic databases (MEDLINE, Embase, Cochrane Library, and PubMed/www.ncbi.nlm.nih.gov).

\section{Results}

Between bacterial extracts we can include two major categories:

Compounds that contain killed bacteria or chemical/mechanical bacterial lysates: OM 85, PMBL

Compounds that contain immunogenic components such as ribosomes and glycoproteins: D53, RU 41740

Bacterial extracts are specific and non-specific stimulating agents, indicated in the prevention and treatment of respiratory infections, particularly in acute and chronic bronchitis, pharyngitis, tonsillitis, laryngitis, rhinitis, sinusitis and otitis. They can also be used as adjuvants in case of infections resistant to common antibiotics or in the treatment of complications [10]. These drugs are able to activate monocytes and macrophages derived from the presence of structures of the bacterial wall, such as protidoglicans or lipopolysaccharide, which interact with the TLR-toll like receptor expressed on the surface of monocytes. This interaction activates monocytes, their differentiation into immature dendritic cells and subsequently in antigen presenting cells. The presentation of antigen on mature dendritic cells induces $\mathrm{T}$ helper function and maturation of $\mathrm{B}$ lymphocytes into plasma cells with subsequent production of antibodies, including the salivary IgA. Such antibodies opsonize bacteria, subsequently phagocyted by granulocytes.
Spisek et al. in their study evaluated the effect of different bacterial immunomodulators on the maturation of immature monocytederived dendritic cells. According to current knowledge about the activation of dendritic cells by microbial products, it is not surprising that lysats or bacterial extracts induce in vitro maturation of dendritic cells, through activation of Toll-like receptor. More surprising is the fact that several immunomodulators induce this activation unevenly. The authors speculate that this may in part be explained by differences in their composition and due to the different production techniques. The results obtained in this study are relevant to understand the mechanism of action of in vivo immunomodulatory drugs, up to now largely unexplained. All these products are administered orally and come to the intestinal lumen, where they can be delivered to dendritic cells localized in the mucosa. Their entry into dendritic cells can be done either via $\mathrm{M}$ cells or by a mechanism that uses the tight junctions between the epithelial cells in the intestinal lumen. The bacterial uptake then results in activation of dendritic cells migrate to secondary lymphoid organs and initiation of an immune response [11].

This immunological effect leads to significant reduction in the number of acute infections and, moreover, in the number and duration of hospitalizations [6] and, as a direct consequence, important reduction in health care costs [7]. The results from the studies conducted to date on bacterial lysates are encouraging.

OM-85 is an immunostimulant extracted from 8 bacterial pathogens of the upper respiratory tract. It contains standardized lyophilized fractions per capsule from the following bacteria: Haemophilus influenzae, Streptococcus pneumoniae, Klebsiella pneumoniae, Klebsiella ozaenae, Staphylococcus aureus, Streptococcus pyogenes, Streptococcus viridans, and Neisseria catarrhalis. OM-85 contains $3,5 \mathrm{mg}$ of standardized lyophilized fractions per capsule from the 8 bacteria.

OM-85 enhances antimicrobial defenses by eliciting IL-12dependent synthesis of IFN- $\gamma$ by $\mathrm{CD}^{+}{ }^{+} \mathrm{T}$ cells [12]. Huber et al. [13] analyzed the immunomodulatory effects of OM-85 in vitro and in vivo and have found that in spleen cell supernatants levels of the $\mathrm{T}_{\mathrm{H}}{ }^{1-}$ associated cytokine IFN- $\gamma$ were increased and levels of the $\mathrm{T}_{\mathrm{H}} 2$ associated cytokine IL- 4 were decreased. The immunoprotective effects of OM-85 might result from its ability to up regulate the $\mathrm{T}_{\mathrm{H}} 1$ response.

Bessler et al. administered OM- 85 by the oral route to mice, to stimulate protective mucosal immunity against influenza virus infection [14]. They distinguished three treatment groups: all animals in the group treated with $1,75 \mathrm{mg}$ OM-85/mouse has survived, compared to $70 \%$ surviving in the group treated with $0,175 \mathrm{mg} / \mathrm{mouse}$ and the untreated control group; treated animals presented with milder clinical symptoms in respect to untreated mices. Additionally, this study suggests that higher dose of OM-85 may have a stimulatory effect. The group treated with the higher dose of OM-85 also showed a lower loss of body weight than the other two groups. These data confirm results from a previous paper published by the same author suggesting that OM-85 enhances the efficacy of influenza vaccine in the mice [15].

Several randomized clinical trials have shown that OM-85 can reduce the number of ARTI of $25-50 \%$ in adults and children [16-21]. However, these studies were designed to demonstrate the preventive effect of OM-85 on AIRT in children with recurrent infections defined by the presence of diagnostic symptoms for at least 48 hours. 
Steurer-Stey et al. [22] in 2007 used a partially meta-analytical approach of published data with OM-85. They concluded that their "sistematic review provides weak evidence that oral immunostimulation with bacterial extracts prevents acute AIRT in children". The 13 studies included in the analyses were considered of low to moderate quality due to limited size of trials, different endpoints and difference in- outcomes definition, occultation of treatment allocation and details about statistical analyses, sponsorship of pharmaceutical companies and conflict of interest with the authors.

More recently Schaad in 2010 published a systematic review on efficacy of OM-85 in the prevention of pediatric ARTI. This review included 22 publications: nine studies were retained as relevant. During the observation period recurrence of ARTI (defined by 3 or more ARTI per 6 months) was $32 \%$ in patients treated with the OM- 85 vs $58,2 \%$ of the placebo treated population, indicating that the active treatment led to $26,2 \%$ fewer patients with recurrent ARTI; a significant reduction in the use of antibiotic therapy was also registered. The NNT was 6.2 considering patients with 1 or more ARTI in a 6 month period; if considering children with 3 or more ARTI, the NNT was 3.8 (Table 1) [23].

\begin{tabular}{|c|c|c|c|c|}
\hline Author (year) & 1 or more ARTI & & 3 or more ARTI & \\
\hline & OM-85 & Placebo & OM-85 & Placebo \\
\hline Schaad et al. (2002) [24] & $103(85.8 \%)$ & $87(87 \%)$ & $39(32.5 \%)$ & $44(44 \%)$ \\
\hline $\begin{array}{l}\text { Gutierrez-Tarango and Berber (2001) } \\
\text { [19] }\end{array}$ & $23(85.2 \%)$ & $28(100 \%)$ & $14(51.9 \%)$ & $23(82.1 \%)$ \\
\hline Jara-Perez and Berber (2000) [17] & $86(86 \%)$ & $100(100 \%)$ & $14(14 \%)$ & $70(70 \%)$ \\
\hline Del Rio et al. (2003) [25] & $2(10 \%)$ & $20(100 \%)$ & $14(70 \%)$ & $19(95 \%)$ \\
\hline Paupe (1990) [21] & $37(60.7 \%)$ & $46(83.6 \%)$ & $19(31.1 \%)$ & $34(61.8 \%)$ \\
\hline Zagar and Löfler-Badzek (1988) [26] & $8(27.6 \%)$ & $9(40.9 \%)$ & $1(3.4 \%)$ & $5(22.7 \%)$ \\
\hline Schaad et al. (1986) [27] & $39(86.7 \%)$ & $48(98 \%)$ & $28(62.2 \%)$ & $28(57.1 \%)$ \\
\hline Gomez-Barreto et al. (1998) [18] & $13(50 \%)$ & $21(70 \%)$ & $8(30.8 \%)$ & $12(40 \%)$ \\
\hline $\begin{array}{l}\text { Pooled } \\
\text { (significance: } P<0.001 \text { ) }\end{array}$ & $311(72.7 \%)$ & $359(88.9 \%)$ & $137(32 \%)$ & $235(58.2 \%)$ \\
\hline Number needed to treat & 6.2 & & 3.8 & \\
\hline Odds ratios OM-85: placebo & $0.33(95 \% \mathrm{Cl} 0.23,0.49)$ & & $0.33(95 \% \mathrm{Cl} 0.25,0.45)$ & \\
\hline \multicolumn{5}{|c|}{ "Robust analysis" after deleting Jara-Perez [17] (2000) as positive 'outlier' \& Schaad [27] (1986) as negative 'outlier': } \\
\hline $\begin{array}{l}\text { Pooled } \\
\text { (significance: } P<0.001 \text { ) }\end{array}$ & $186(65.7 \%)$ & $211(82.7 \%)$ & $95(33.6 \%)$ & $137(53.7 \%)$ \\
\hline
\end{tabular}

Table 1: Number of patients with ARTI ( $\geq 1$ or $\geq 3$ ARTI/6 months), per trial \& treatment (Schaad et al. [23])

The mean number of ARTI in the previous year was similar in patients treated with OM-85 and placebo in most of the studies, but clinical trials differ in age of treated patients and in therapeutic regimens.

In addition, the incidence of minor adverse events (mainly gastrointestinal or cutaneous findings) was $17,7 \%$ for the OM-85 treated group and $18,2 \%$ for those treated with placebo. Serious adverse events were reported in $1 \%$ vs $0.5 \%$, withdrawals due to adverse event in $1.3 \%$ vs $0.7 \%$, respectively. Anyhow, no causal relationship has been established for any of these adverse events [23].

Razi et al. [28] in 2010 conducted a study which included children from 1 to 6 years old with 3 or more acute wheezing attacks induced by respiratory tract illness in the previous 6 months. This study was a randomized, double-blind, placebo-controlled, parallel-group study with OM-85 in patients with recurrent wheezing. It was conducted from August 2007 to September 2008 in a hospital setting in Ankara, Turkey. The children received 1 capsule of OM- 85 or placebo per day for 10 consecutive days in each of 3 months. Patients were evaluated monthly and whenever they had respiratory tract symptoms. If patients had a wheezing attack, the number of days to clinical cure, the number of days that $\beta_{2}$-agonists and oral steroids were used and the number and duration of hospitalizations were recorded.

They demonstrated that treatment with OM-85 significantly reduced the incidence of acute virus-provoked wheezing attacks: the main factor associated with the lowering in wheezing attacks was a reduction in ARTI. OM-85 significantly reduced the mean incidence of ARTI and acute nasopharyngitis by $31.5 \%$ and $37.9 \%$, respectively. Moreover, the decrease in the number and duration of wheezing attacks after administration of OM-85 could have positive secondary effects, such as reduced use of short-acting $\beta_{2}$-agonists or systemic/ 
topical corticosteroids, fewer visits to emergency services and lost work days by parents.

Data from the literature are globally encouraging but more clinical studies are necessary to allow some routine preventive treatment recommendation.

PMBL is a polyvalent mechanical bacterial lysate obtained by mechanical lysis of 48 billion bacteria commonly responsible for upper and lower respiratory tract infections (S. aureus, $S$. pyogenes, $K$. pneumoniae, $S$. viridans, $K$. ozaenae, $H$. influenzae serotype $B, M$. catarrhalis, and $S$. pneumoniae).

It is produced through a process that preserves the structure of the bacterial antigens and consists in mechanical lysis, without the use of any chemical products to denature the antigenic structure of bacteria. This ensures a preparation having excellent antigenic properties. In in vitro study, it has been demonstrated that the particulate fraction of the antigens was more efficient than the soluble antigens in inducing maturation of the dendritic cells.

A recent publication has shown that PMBL, thanks to its preserved antigenic structures, is 10-100 times more effective than chemical lysates to trigger an immune response [29]. This could explain why bacterial lysates obtained by mechanical lysis are more powerful than chemical lysates for prophylaxis [29]. Indeed, although OM-85 and Polyvalent mechanical bacterial lysate (PMBL) are bacterial lysate made from the same wide range pathogenic bacteria, they are substantially different: OM-85 is the product of alkaline proteolysis, a process that may determine protein denaturation, with a consequent lower immunogenicity of the antigens themselves, whereas PMBL is produced through mechanical lysis by increasing pressure on bacterial cell walls, which ensures a preparation having excellent antigenic properties. This combined with the large variety of pathogens used and its sublingual mode of administration, confer to PMBL optimal immunogenic activity [29].

The development of an effective mucosal vaccine has potentially a number of advantages, including lowering of bacterial carriage levels in the respiratory tract, decreasing the incidence and severity of exacerbations, decreasing hospitalization due to exacerbations, and reducing the need for antibiotics. In particular sublingual administration guarantees effective protection of the respiratory mucosae, which represent the first barrier to infection, making it possible to bypass the gastroenteric tract. This administration has a downside: the compliance of the patient. In fact, for optimal administration of PMBL, the preparation must be kept under the tongue for at least 5-7 minutes. It's simple to think that this procedure can be conducted only with children who are old enough (i.e. 5 years) and sufficiently motivated to understand the need for respecting this procedure [30].

It is recommended in the prevention of acute, sub-acute, recurrent or chronic infections of the upper and lower airways in adults and children [31].

PMBL have already shown efficacy in the prophylaxis of exacerbations in Chronic Obstructive Pulmonary Disease (COPD), a state characterized by airflow limitation that is preventable and treatable, but not fully reversible and usually progressive [32].

The clinical data available in COPD patients indicated a positive trend in term of reduction of acute exacerbations rate and duration, beneficial effect on symptoms, reduction in antibiotics use, hospitalization time and possibility to improve the patient's quality of life [8].

The literature supports the possibility that bacterial immunostimulants might be considered as possible adjuvants in the prevention of influenza episodes, and prolongs antibody response to influenza vaccine strains. In fact, some data indicate interesting synergic effects of bacterial extracts in extending the immunological response to influenza vaccine in COPD patients [33]

Data obtained in vitro, by using the cytofluorimetry technique, have proven that PMBL can induce the activation of dendritic cells [34] and, in general, it can promote the activation of innate immune response and specific ones both in vitro and in vivo [35].

Moreover, clinical trials [36] clearly demonstrate that this use decreases the number of respiratory infections. It indicates that the use of PMBL might be recommended for also preventing influenza episodes.

A pilot study involving 89 children aged 10 months-10 years showed that the treatment with PMBL significantly reduced the rate of recurrent respiratory infections, not only among the same children in relation to the previous winter, but also in comparison with untreated children during the same winter (mean number of infective episodes per patient 7.84 vs $4.78, \mathrm{P} \leq 0.05$ with $\mathrm{PMBL} ; 6.78$ vs $4.78, \mathrm{P} \leq 0.05$ without PMBL); also phlogosis indexes (PCR, plasma mucoprotein and white blood cell) were significantly lower in the treated group, and the values of B-lymphocytes were found to be increased (9.9 with PMBL vs 5.9 without PMBL) [30].

Although the results of several studies are encouraging, it would be worthwhile to carry out further trials that will be well-designed in term of blinding and randomization procedures and include a higher number of patients selected.

Also ribosomal immunotherapeutic agent may prevent ARTI in children. The product name D53 contains Klebsiella pneumoniae protidoglicans and ribosomal extracts belonging to 4 different bacterial species, such as S.pneumoniae, K. pneumoniae, S. pyogenes, $H$. influenzae. It stimulates production of specific antibodies against the four bacterial strains included in the compound and, also, stimulates non-specific immunity; particularly, it stimulates dendritic cells and enhances expression of surface molecules involved in the antigen presentation, induces TLR-2 expression, promotes T-cell differentiation and regulates the relationship Th1/Th2, induces IgA production, stimulates pro-inflammatory cytokines and of free radicals production and, finally, stimulates phagocytosis and NK cell activity [37].

In 2006 Bousquet and Fiocchi [9] reviewed the literature concerning clinical evidence of D53 efficacy and found that this product reduced the number and duration of upper or lower ARTI, fever episodes, number of antibacterial treatments; a smaller number of lost school days or parent absenteeism from work was also registered. The 12 clinical trials on children $(n=1215)$ selected were analyzed in three categories based on pathology: ENT (ear, nose and throat) infections, mixted ENT/brocopulmonary infections and acute otitis media. In all three categories the administrations of ribosomal immunotherapy was statistically more effective than placebo in preventing recurrence of infectous episodes (between 27\% and 68\% fewer ENT infections, between $32 \%$ and $61 \%$ fewer ENT/ brocopulmonary infections and between $10 \%$ and $53 \%$ fewer otitis media vs placebo). 
Adverse events occurred very rarely and their frequency was fewer to that reported in placebo groups (1.6\% in D53 group's vs $3.2 \%$ in the placebo groups): mostly, adverse events consisted of fever, cutaneous rashes and otorhinolaryngologic. In conclusion, this review showed that ribosomal agent could be effective in preventing ARTI in children [9].

Also cited in the scientific literature is the molecule RU 41740. It's a glycoprotein extracted from Klebsiella pneumoniae that stimulates immune response in the mucosal associated lymphoid tissue, alveolar macrophages and dendritic cells activity and phagocytosis and, finally, enhances B-cell antibody production.

The preventive treatment of mice with RU 41740 leads to a fall of pro-inflammatory cytokines and an increase of IgG antibodies. Using a mouse model of experimental infection, Nimier et al. [38] demonstrated that the number of mice alive after infection of Klebsiella pneumoniae and E. aerogenes increased by 40 and $30-40 \%$, respectively, in the RU 41740-treated groups compared to the control groups. In these experimental conditions no significant protection was conferred by RU $4 \mathrm{I} 740$ on mice infected with the gram-positive specie S. warneri. However, oral administration of $100 \mathrm{mg} / \mathrm{kg} \mathrm{RU} 41740$ resulted in a weak but significant increase in the survival rate of mice infected by the intracellular gram-positive bacterium $\mathrm{L}$. monocytogenes: four days after infection, the survival rate was already increased in when RU 41740 was administered at a lower dose $(20 \mathrm{mg} /$ $\mathrm{kg}$ ). These results suggest that oral treatment with RU 41740 results not only in a specific protective effect against gram-negative Enterobacteriaceae and intracellular gram-positive bacteria [38].

In the literature, there are two clinical trials available, dating 1986 and 1990, conducted in France. In a first double-blind, placebocontrolled study, 45 children aged 1-13 years with a history of ARTI: 20 children received RU 41740 while 22 patients received placebo for 8 days/month for 3 months. Data analysis did not show a statistically significant difference between two groups $(p=0.09)$. The drug's protective effect was demonstrated by the significant reduction in the duration of infections (10 days vs 34 ) and antimicrobial therapy (10 days vs 20) over a six-month period in trested patients. Clinical and biologic tolerance was very satisfactory [39].

In the second one, Bellon et al. administered the product once a day, 8 days/month for 3 months to 3008 children with a history of ARTI, aged 1-16 years. Results were encouraging: $75.3 \%$ of the children had either no infection or only one infection during the 3 months follow-up. Tolerance was good in $98.6 \%$ of cases, the remaining percentage presented mild to minor side-effects.

Although the available results are encouraging, further studies are necessary to confirm the efficacy of the product.

\section{Conclusion}

Data from the literature regarding the use of some immunostimulants in the prevention of ARTI are encouraging in terms of disease incidence and duration reduction. This analysis may help the practitioners in identifying the best candidates for immunostimulant therapy and the role of this therapy and her positive effects in the prevention of ARTI.

Overall, bacterial lysates and extracts may reduce the incidence of ARTI of about $40 \%$ in toddlers (2-5 years), school boys (6-12 years) and children with high incidence ARTI (eg children living in orphanages) [21]. Differences in the composition of bacterial extracts and the different technology of their production could explain the variability in their effectiveness [7]. More attention is allocated to treatment with OM-85, the main formulation in terms of scientific evidence. Exploratory analysis indicates that efficacy is more pronounced in patients at hight risk of recurrent ARTI; the benefit of OM-85 as compared to placebo was notoriously greater in children with a history of very frequent ARTI.

Future studies should provide details of the ARTI occurring during the observation period employing a standardized clinical classification, because so far both the observation period for the collection of data, both the recurrence of infectious episodes were set arbitrarily. Therefore we suggest to start from the definition of ARTI quoted at the beginning that "are characterized by the onset of more than six respiratory infections per year or more than one upper respiratory infection per month or more than three lower respiratory infections during the period of maximum exposure (September to April), in a child that does not suffer pathological conditions to justify respiratory infections recurrence [2].

At the time of this review, we did not find any study regarding healthy children: the studies presented were designed to demonstrate the effectiveness of bacterial extracts or synthetic immunomodulators in reducing episodes of acute respiratory infections in children suffering from ARTI only.

The strengthening of pre-clinical and clinical research will help provide new answers and new evidence in order to allow routine recommendation of bacterial extracts and synthetic immunomodulators prescription for ARTI prevention in children.

\section{References}

1. Arrighi A (2000) Evaluation of clinical efficacy in a homotoxicologic protocol for prevention of recurrent respiratory infections in pediatrics. La medicina biologica: 13-21.

2. Cuppari C, Manti S, Salpietro A, Colavita L, Valenti S De Vivo D, et al. (2012) New Hypothesis: correlation between flogosis allergic minimum persistent (P.A.M.P.) and recurrent respiratory infections. Bi-Monthly Journal of Pediatrics 1

3. Caramia G, Clemente E, Solli R, Mei V, Cera R, et al. (1994) Efficacy and safety of Pidotimod in the treatment of recurrent respiratory infections in children, Arzneim -Forsch/Drug Res 44: 1480-1484.

4. Dagan R, Sikuler-Cohen M, Zamir O, Janco J, Givon-Lavi N, et al. (2001) Effect of a conjugate pneumococcal vaccine on the occurrence of respiratory infections and antibiotic use in day-care center attendees. Pediatr Infect Dis J 20: 951-958.

5. Esposito S, Marchisio P, Cavagna R, Gironi S, Bosis S, et al. (2003) Effectiveness of influenza vaccination of children with recurrent respiratory tract infections in reducing respiratory-related morbidity within the households. Vaccine 21:3162-3168.

6. Donaldson GC, Seemungal TA, Bhowmik A, Wedzicha JA (2002) Relationship between exacerbation frequency and lung function decline in chronic obstructive pulmonary disease. Thorax 57: 847-852.

7. Miravitlles M, Mayordomo C, Artés M, Sánchez-Agudo L, Nicolau F, et al. (1999) Treatment of chronic obstructive pulmonary disease and its exacerbations in general practice. EOLO Group. Estudio Observacional de la Limitación Obstructiva al Flujo aEreo. Respir Med 93: 173-179.

8. Cazzola M, Noschese P, Di Perna F (2009) Value of adding a polyvalent mechanical bacterial lysate to therapy of COPD patients under regular treatment with salmeterol/fluticasone. Therapeutic Advances in Respiratory Disease 3: 59-63.

9. Bousquet J, Fiocchi A (2006) Prevention of recurrent respiratory tract infections in children using a ribosomal immunotherapeutic agent: a clinical review. Paediatr Drugs 8: 235-243. 
10. Braido F, Tarantini F, Ghiglione V, Melioli G, Canonica GW (2007) Bacterial lysate in the prevention of acute exacerbation of COPD and in respiratory recurrent infections. Int J Chron Obstruct Pulmon Dis 2: 335-345.

11. Spisek R, Brazova J, Rozkova D, Zapletalova K, Sediva A, et al. (2004) Maturation of dendritic cells by bacterial immunomodulators. Vaccine 22: 2761-2768.

12. Byl B, Libin M, Gérard M, Clumeck N, Goldman M, et al. (1998) Bacterial extract OM85-BV induces interleukin-12-dependent IFNgamma production by human CD4+ T cells. J Interferon Cytokine Res 18: $817-821$.

13. Huber M, Mossmann H, Bessler WG (2005) Th1-orientated immunological properties of the bacterial extract OM-85-BV. Eur J Med Res 10: 209-217.

14. Bessler WG, Vor dem Esche U, Masihi N (2010) The bacterial extract OM-85 BV protects mice against influenza and Salmonella infection. Int Immunopharmacol 10: 1086-1090.

15. Bessler WG, Huber M, Baier W (1997) Bacterial cell wall components as immunomodulators--II. The bacterial cell wall extract OM-85 BV as unspecific activator, immunogen and adjuvant in mice. Int J Immunopharmacol 19: 551-558.

16. Mauël J (1994) Stimulation of immunoprotective mechanisms by OM-85 BV. A review of results from in vivo and in vitro studies. Respiration 61 Suppl 1: 8-15.

17. Jara-Perez JV, Berber A (2000) Primary prevention of acute respiratory tract infections in children using a bacterial immunostimulant: a doublemasked, placebo-controlled clinical trial. Clin Ther 22: 748-759.

18. Gómez Barreto D, De la Torre C, Alvarez A, Faure A, Berber A (1998) [Safety and efficacy of OM-85-BV plus amoxicillin/clavulanate in the treatment of subacute sinusitis and the prevention of recurrent infections in children]. Allergol Immunopathol (Madr) 26: 17-22.

19. Gutiérrez-Tarango MD, Berber A (2001) Safety and efficacy of two courses of OM-85 BV in the prevention of respiratory tract infections in children during 12 months. Chest 119: 1742-1748.

20. Collet JP, Ducruet T, Kramer MS, Haggerty J, Floret D, et al. (1993) Stimulation of nonspecific immunity to reduce the risk of recurrent infections in children attending day-care centers. The Epicrèche Research Group. Pediatr Infect Dis J 12: 648-652.

21. Paupe J (1991) Immunotherapy with an oral bacterial extract (OM-85 BV) for upper respiratory infections. Respiration 58: 150-154.

22. Steurer-Stey C, Lagler L, Straub DA, Steurer J, Bachmann LM (2007) Oral purified bacterial extracts in acute respiratory tract infections in childhood: a systematic quantitative review. Eur J Pediatr 166: 365-376.

23. Schaad UB (2010) OM-85 BV, an immunostimulant in pediatric recurrent respiratory tract infections: a systematic review. World Pediatr 6: 5-12

24. Schaad UB, Mütterlein R, Goffin H; BV-Child Study Group (2002) Immunostimulation with OM-85 in children with recurrent infections of the upper respiratory tract: a double-blind, placebo-controlled multicenter study. Chest 122: 2042-2049.

25. Del Rio Navarro BE, Luis Siemra-Monge JJ, Berber A, Torres-Alcantara S, Avila-Castañon L, et al. (2003) Use of OM-85 BV in children suffering from recurrent respiratory tract infections and subnormal IgG subclass levels. Allergol et Immunopathol 31: 7-13.

26. Zagar S, Löfler-Badzek D (1988) Broncho-Vaxom in children with rhinosinusitis: a double-blind clinical trial. ORL J Otorhinolaryngol Relat Spec 50: 397-404.

27. Schaad UB, Farine JC, Fux T (1986) [Prospective placebo-controlled double-blind study using a bacterial lysate in infections of the respiratory tract and ENT region in children]. Helv Paediatr Acta 41: 7-17.

28. Razi CH, Harmancı K, Abacı A, Özdemir O, Hızlı S, et al. (2010) The immunostimulant OM-85 BV prevents wheezing attacks in preschool children. J Allergy Clin Immunol 126: 763-769.

29. Morandi B, Agazzi A, D’Agostino A, Antonini F, Costa G, et al. (2011) A mixture of bacterial mechanical lysates is more efficient than single strain lysate and of bacterial- derived soluble products for the induction of an activating phenotype in human dendritic cells. Immunol Lett 138: 86-91.

30. Rosaschino F, Cattaneo L (2004) Strategies for optimizing compliance of paediatric patients for seasonal antibacterial vaccination with sublingually administered polyvalent mechanical bacterial lysates (PMBL). Acta Biomed Ateneo Parmense 75: 171-178.

31. Cazzola M, Rogliani P, Curradi G (2008) Bacterial extracts for the prevention of acute exacerbations in chronic obstructive pulmonary disease: a point of view. Respir Med 102: 321-327.

32. Cazzola M, Anapurapu S, Page CP (2012) Polyvalent mechanical bacterial lysate for the prevention of recurrent respiratory infections: a meta-analysis. Pulm Pharmacol Ther 25: 62-68.

33. Centanni S, Pregliasco F, Bonfatti C, Mensi C, Tarsia P, et al. (1997) Clinical efficacy of a vaccine-immunostimulant combination in the prevention of influenza in patients with chronic obstructive pulmonary disease and chronic asthma. J Chemother 9: 273-278.

34. Cangemi G, Morandi B, Ferlazzo G, Melioli G (2004) The maturation of dendritic cells mediated by a polivalent mechanical bacterial lysate (PBML) results in the secretion of cytokines inducing an anti-bacterial polarization of the locoregional immune-response. Eur Respir J.

35. Fuggetta MP, Lanzilli G (2007) Attivazione delle risposte immunitarie mediante vaccini batterici Europ Respir News 15: 69-77.

36. Rossi S, Tazza R (2004) Efficacy and safety of a new immunostimulating bacterial lysate in the prophylaxis of acute lower respiratory tract infections. A randomised, open, controlled clinical trial. Arzneimittelforschung 450-456.

37. Rozy A, Chorostowska-Wynimko J (2008) Bacterial immunostimulants-mechanism of action and clinical application in respiratory diseases. Pneumonol Alergol Pol 76: 353-359.

38. Nimier K, Wolff F, Allouch PY, Guy-Grand D, Bloy C (1999) Protective effects of RU 41740, a bacterial immunomodulator, against experimental infections: induction of cytokine and immunoglobulin release in mice after oral administration. Int J Immunopharmacol 21: 561-574

39. Paupe J, Paupe G (1986) [Biostim prevention of recurrent respiratory infections in children. A double-blind versus placebo study]. Ann Pediatr (Paris) 33: 843-845. 